\title{
Genomic imbalances detected by array-CGH in patients with syndromal ocular developmental anomalies
}

\author{
Andrée Delahaye ${ }^{\star, 1,2,3,15}$, Pierre Bitoun ${ }^{4,15}$, Séverine Drunat ${ }^{5}$, Marion Gérard-Blanluet ${ }^{5}$, Nicolas Chassaing ${ }^{6}$, \\ Annick Toutain $^{7}$, Alain Verloes ${ }^{3,5,8}$, Frédérique Gatelais ${ }^{9}$, Marie Legendre ${ }^{10}$, Laurence Faivre $^{11}$, Sandrine Passemard ${ }^{3,5,8}$, \\ Azzedine Aboura $^{5}$, Sophie Kaltenbach ${ }^{5,12}$, Samuel Quentin ${ }^{13}$, Céline Dupont ${ }^{1,5}$, Anne-Claude Tabet ${ }^{5}$, \\ Serge Amselem 9 , Jacques Elion ${ }^{3,5,8,14}$, Pierre Gressens ${ }^{3,8}$, Eva Pipiras ${ }^{1,2,3,16}$ and Brigitte Benzacken ${ }^{1,2,3,5,16}$
}

In 65 patients, who had unexplained ocular developmental anomalies (ODAs) with at least one other birth defect and/or intellectual disability, we performed oligonucleotide comparative genome hybridisation-based microarray analysis (array-CGH; $105 \mathrm{~A}$ or $180 \mathrm{~K}$, Agilent Technologies). In four patients, array-CGH identified clinically relevant deletions encompassing a gene known to be involved in ocular development (FOXC1 or OTX2). In four other patients, we found three pathogenic deletions not classically associated with abnormal ocular morphogenesis, namely, del(17)(p13.3p13.3), del(10)(p14p15.3), and del(16)(p11.2p11.2). We also detected copy number variations of uncertain pathogenicity in two other patients. Rearranged segments ranged in size from 0.04 to $5.68 \mathrm{Mb}$. These results show that array-CGH provides a high diagnostic yield (15\%) in patients with syndromal ODAs and can identify previously unknown chromosomal regions associated with these conditions. In addition to their importance for diagnosis and genetic counselling, these data may help identify genes involved in ocular development. European Journal of Human Genetics (2012) 20, 527-533; doi:10.1038/ejhg.2011.233; published online 11 January 2012

Keywords: ocular developmental anomaly; array-CGH; OTX2; FOXC1; 16p11.2 deletion; YWHAE

\section{INTRODUCTION}

Ocular developmental anomalies (ODAs) are severe structural defects of the eye caused by disruption of the complex process of ocular morphogenesis during early gestation. ${ }^{1}$ ODAs occur in about 1 in 3000 to 4000 neonates and have been estimated to account for at least $25 \%$ of cases of childhood visual impairment worldwide. ${ }^{2,3}$ ODAs include anophthalmia, microphthalmia, coloboma (failure of the optic fissure to close), congenital cataract (opacity of the lens fibres), and dysgenesis of the anterior segment (iris and cornea). These anomalies can occur separately or in combination and can be accompanied with other birth defects and/or with intellectual disability. The broad clinical spectrum of syndromal ODAs reflects the complexity of the pathways involved in ocular development. Many genes are known to be involved in ocular development, including several genes initially identified in chromosomal rearrangements. ${ }^{4}$

Chromosomal abnormalities are found in 7.7 to $10 \%$ of neonates with ocular anomalies and other birth defects. ${ }^{5,6}$ The introduction of microarray technology has shown a very high rate of rearrangements undetectable with standard or high-resolution karyotyping. Submicroscopic copy number variations (CNVs) account for a substantial proportion of normal and pathogenic genetic variation in humans. ${ }^{7}$
Few studies have specifically addressed the role for submicroscopic chromosomal imbalances in ODAs. In a case-series study of 32 patients with non-syndromal anophthalmia, microphthalmia, or coloboma, a single causal deletion was found, suggesting a limited causal role for CNVs in non-syndromal ODAs. ${ }^{8}$ Another study found pathogenic CNVs in $5(13 \%)$ of 37 patients with ocular birth defects. ${ }^{9}$

The objective of this study was to determine the prevalence of genomic imbalances identified using comparative genomic hybridisation-based microarray analysis (array-CGH) in patients with syndromal ODAs.

\section{PATIENTS AND METHODS}

Informed consent to participation was obtained from all patients and/or parents before study inclusion. Parents also gave informed consent for their own tests.

\section{PATIENTS}

A total of 65 unrelated patients (42 males and 23 females) were included. They were born to non-consanguineous parents and had unexplained syndromal ODAs with normal karyotypes. All patients were examined by experienced ophthalmologists and clinical

\footnotetext{
${ }^{1}$ AP-HP, Hôpital Jean Verdier, Service d'Histologie, Embryologie, et Cytogénétique, Bondy, France; ²Université Paris-Nord, Paris 13, UFR SMBH, Bobigny, France; ${ }^{3}$ Inserm, U676, Paris, France; ${ }^{4}$ AP-HP, Hôpital Jean Verdier, Consultations de génétique médicale, Service de Pédiatrie, Bondy, France; ${ }^{5}$ AP-HP, Hôpital Robert Debré, Département de Génétique, Paris, France; ${ }^{6} \mathrm{CHU}$ Toulouse, Hôpital Purpan, Service de Génétique Médicale, Toulouse, France; ${ }^{7} \mathrm{CHU}$ de Tours, Hôpital Bretonneau, Département de Génétique, Tours, France; ${ }^{8}$ Université Paris Diderot, Faculté de Médecine, Paris, France; ${ }^{9} \mathrm{CHU}$ d'Angers, Service d'endocrinologie diabétologie pédiatrique, Angers, France; ${ }^{10}$ Inserm, U933, Université Paris 6 , AP-HP, Hôpital Armand-Trousseau, Paris, France; ${ }^{11} \mathrm{CHU}$ de Dijon, Centre de Génétique, Dijon, France; ${ }^{12}$ AP-HP, Hôpital Necker-Enfants-Malades, Service de Cytogénétique, Université Paris Descartes, Paris, France; ${ }^{13} \mathrm{AP}-\mathrm{HP}$, Hôpital Saint-Louis, Plateforme génomique, Institut Universitaire d'Hématologie, Paris, France; ${ }^{14}$ Inserm, U763, Paris, France ${ }^{*}$ Correspondence: Dr A Delahaye, AP-HP, Hôpital Jean Verdier, Service d'Histologie, Embryologie, et Cytogénétique, Avenue du 14 Juillet, 93140 Bondy, France. Tel: +33 148 026674 ; Fax: +33 148 026737; E-mail: andree.delahaye@jvr.aphp.fr

${ }^{15}$ These authors contributed equally to this work.

${ }^{16}$ These authors contributed equally to this work.
}

Received 15 June 2011; revised 11 November 2011; accepted 15 November 2011; published online 11 January 2012 
geneticists, and had negative routine diagnostic work-up results. There were 38 patients with micro-anophthalmia and coloboma, 7 with optic nerve hypoplasia, 2 with aniridia, 8 with anterior segment anomalies, 8 with congenital cataract, and 2 with other ocular defects (Duane syndrome and buphthalmos, respectively). Only patients with intellectual disability and/or at least one extraocular birth defect were included. Individual patient characteristics are reported in Supplementary Table 1.

\section{Array-CGH}

Blood samples were obtained from the study patients and, when possible, from their parents. Genomic DNA was isolated from blood samples using standard protocols. Oligonucleotide array-CGH was performed using the Human Genome CGH Microarray Kit 105A or SurePrint G3 Human CGH Microarray Kit, $4 \times 180 \mathrm{~K}$ (Agilent Technologies, Santa Clara, CA, USA). In the 105A kit, the arrays include a total of 105750 probes, with an overall median probe spacing of $22 \mathrm{~kb}$, and in the $180 \mathrm{~K}$ kit a total of 180880 probes, with an overall median probe spacing of $13 \mathrm{~kb}$. Experiments were performed according to the standard Agilent protocol (Agilent Oligonucleotide Array-Based CGH for Genomic DNA Analysis, version 6.3). Commercially available genomic DNA (Promega, Madison, WI, USA) was used as the control. Hybridised slides were scanned with a microarray scanner (G2505B SureScan High-Resolution Technology Agilent), and the image data were extracted and converted to text files using Agilent Feature Extraction software. The data were graphed and analysed using Agilent CGH Analytics software (statistical algorithm: ADM-2; sensitivity threshold: 6.1). Only gains or losses that encompassed at least three consecutive oligomers on the array were considered. CNVs previously identified in unaffected individuals according to the Database of Genomic Variants (http://projects.tcag.ca/variation/) were excluded. The validation method was chosen based on the imbalance type (deletion or duplication), size, and sample availability (DNA and/or metaphase spreads). Then, the clinical relevance of observed chromosomal aberrations was estimated according to data found in the scientific literature and databases for each of the regions and genes involved, using the DECIPHER database (http://www.sanger.ac.uk/ PostGenomics/decipher/) for known microdeletion and microduplication syndromes and the Online Mendelian Inheritance in Man (OMIM, www.ncbi.nlm.nih.gov/Omim/getmorbid.cgi) for known disease-causing genes, gene functions, and inheritance patterns. Whenever possible, to discriminate between de novo and inherited anomalies, the parents were tested using fluorescence in situ hybridisation (FISH), multiplex ligation-dependent probe amplification (MLPA), or real-time quantitative PCR technology (qPCR). When there was a family history of ODA or X-linked anomaly, additional family members were studied to evaluate the familial segregation of the inherited anomalies. DNA copy alterations were considered possibly pathogenic when they involved regions known to be associated with microdeletion or microduplication syndromes, involved known dosage-sensitive genes, involved known eye development genes, consisted in de novo imbalances, or exhibited a pattern of family segregation consistent with pathogenicity.

\section{Fluorescence in situ hybridisation}

FISH was performed using standard protocols with commercially available probes. BAC clones from the RPCI human BAC library were selected in the chromosomal region of interest using the UCSC Genome Browser (http://genome.cse.ucsc.edu). DNA was labelled with Spectrum GreenTM-11-dUTP or Spectrum OrangeTM-11-dUTP (Vysis, Downers Grove, IL, USA) by nick translation, using a commercial kit (Roche Diagnostics GMBH; http://www.rochediagnostics.com) according to the manufacturer's instructions. All BAC clones used for confirmation are described in the online Supplementary Information file (Supplementary Table 2).

\section{Multiplex ligation-dependent probe amplification}

The microdeletion syndrome-specific MLPA kit SALSA P297-B1 was used with MRC-Holland reagents (MRC-Holland, Amsterdam, the Netherlands) according to the manufacturer's protocol. Amplification products were analysed using capillary electrophoresis in the DNA Analyser 3130XL and GeneMapper software v3.7 (Applied Biosystems, Life Technologies, Carlsbad, CA, USA). This kit contains probes targeting the $16 \mathrm{p} 11.2$ region (MAZ, MVP, HIRIP3, DOC2A, and MAPK3 genes).

\section{Real-time qPCR}

Real-time qPCR was performed on a StepOnePlus Real-Time PCR System (Applied Biosystems, Life Technologies) with fluorescent SYBR Green dye (Power SYBR green PCR master mix, Applied Biosystems, Life Technologies). Gene-specific primers for the target gene and endogenous control genes (F9 and PTEN) were designed using Primer Express Software (Applied Biosystems, Life Technologies). The BLAST program from the NCBI browser (http://www.ncbi.nlm.nih.gov/BLAST) was used for in silico specificity analysis. Amplification efficiencies were calculated based upon the generation of standard curves using serial dilutions of genomic DNA. Assays with amplification efficiencies between 85 and 120\% were considered acceptable. Each sample was run in triplicate for target gene quantification compared with endogenous control genes. Data were processed using the StepOne software v.2 (Applied Biosystems, Life Technologies), with the comparative $\Delta \Delta$ threshold cycle-number method. ${ }^{10}$ All the primers and probes used for qPCR are described in the online Supplementary Information file (Supplementary Table 2).

\section{Microsatellite analysis}

Microsatellites were selected from UCSC Genome Browser microsatellites or simple repeat tracks, and primers were designed using the NCBI Primer-BLAST program. For a single reaction, a master mix of $2.5 \mu \mathrm{l}$ $10 \times$ PCR buffer with $25 \mathrm{~mm} \mathrm{MgCl} 2,1 \mu \mathrm{l} 5 \mathrm{~mm}$ dNTP, $0.125 \mu \mathrm{l}$ AmpliTaq Gold enzyme (Applied Biosystems, Life Technologies), $0.25 \mu \mathrm{l} 20 \mathrm{pM}$ primers (forward and reverse), and $16375 \mu$ l sterile $\mathrm{H} 2 \mathrm{O}$ was prepared. A

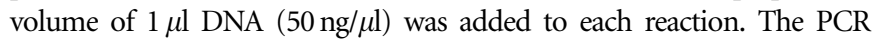
reaction was run in Eppendorf Thermocyclers (Eppendorf, Hamburg, Germany) using the following conditions: hot start at $95^{\circ} \mathrm{C}$ for $10 \mathrm{~min}$, $95^{\circ} \mathrm{C}$ for $10 \mathrm{~s}, 50^{\circ} \mathrm{C}$ for $10 \mathrm{~s}$ for 36 cycles, followed by a final extension step at $72{ }^{\circ} \mathrm{C}$ for $4 \mathrm{~min}$. Samples were analysed on an ABI $3730 \mathrm{XL}$ DNA sequencing analyser and processed using GeneMapper 3.7 software (Applied Biosystems, Life Technologies).

\section{RESULTS}

\section{Molecular karyotyping}

Array-CGH identified 15 DNA CNVs involving segments larger than $100 \mathrm{~kb}$ in 14 patients (Table 1). These alterations were consistently confirmed by FISH, MLPA, or qPCR. The altered segments ranged in size from 0.04 to $5.68 \mathrm{Mb}$ (Figure 1). In four patients, we identified clinically relevant deletions encompassing a gene known to be involved in ocular development (FOXC1 or OTX2; Table 1, patient no. 1-4). In four other patients, three pathogenic deletions not classically associated with ODAs were found, namely, $\operatorname{del}(10)(\mathrm{p} 14 \mathrm{p} 15.3)$, $\operatorname{del}(17)(13.3 p 13.3)$, and $\operatorname{del}(16)(\mathrm{p} 11.2 \mathrm{p} 11.2)$ (Table 1, patient no. 5-8). Microsatellite analyses showed that the deletions were de novo and paternally derived for patient no. 1, 2, and 5, and de novo 
Table 1 DNA copy alterations identified using array-CGH

\begin{tabular}{|c|c|c|c|c|c|c|}
\hline Patient & Imbalance & Size $(M b)$ & ISCN description ${ }^{\mathrm{a}}$ & Inheritance & Major genes involved & DECIPHER patient \\
\hline \multicolumn{7}{|c|}{ Possibly pathogenic chromosomal anomalies } \\
\hline Patient 1 & Del & 5.68 & $6 p 25.1 p 25.3(107,883-5,684,125) \times 1$ & Pat (dn) & FOXC1 & PAR251592 \\
\hline \multirow[t]{2}{*}{ Patient 3} & Del & 0.61 & $4 q 34 q 34(176,398,264-177,004,339) \times 1$ & Pat (inh) & GPM6A & PAR251586 \\
\hline & Del & 2.25 & $14 q 22.2 q 23.1(54,287,767-56,543,234) \times 1$ & Mat (inh) & OTX2, GCH1 & \\
\hline Patient 4 & Del & 0.04 & $11 \mathrm{p} 15.4 \mathrm{p} 15.4(3,765,195-3,809,332) \times 1$ & $U$ & RHOG & PAR254661 \\
\hline Patient 6 & Del & 0.5 & 17p13.3p13.3(1,105,199-1,605,301)x1 & $u$ & YWHAE & PAR251562 \\
\hline Patient 7 & Del & 0.6 & $16 p 11.2 p 11.2(29,500,084-30,106,254) \times 1$ & Mat (dn) & SEZ6L2 & PAR251593 \\
\hline Patient 8 & Del & 0.55 & $16 p 11.2 p 11.2(29,560,300-30,106,254) \times 1$ & Non-mat & SEZ6L2 & PAR256688 \\
\hline Patient 9 & Dup & 0.18 & $11 p 15.4 p 15.4(7,283,552-7,466,165) \times 3$ & Pat (inh) & SYT9, OLFMLI & PAR251548 \\
\hline Patient 10 & Del & 0.36 & $20 p 12.1 p 12.1(15,003,653-15,360,378) \times 1$ & $U$ & MACROD2 & PAR251588 \\
\hline Patient 14 & Del & 0.2 & $8 p 23.2 p 23.3(3,079,016-3,276,030) \times 1$ & Pat (inh) & CSMD1 & PAR251596 \\
\hline
\end{tabular}

Abbreviations: Del, deletion; dn, de novo; Dup, duplication; inh, inherited; ISCN, International System for Human Cytogenetic Nomenclature (2009); mat, maternal; pat, paternal; U, unavailable. aOn NCBI human genome Build 36 (UCSC hg18).

Bold denotes gene known to be involved in ocular development.

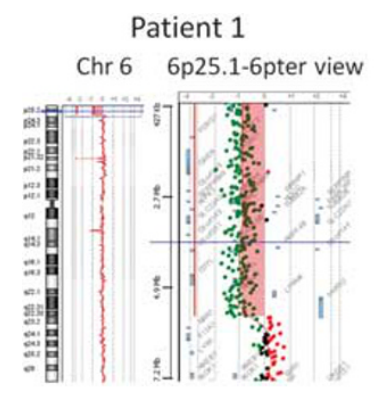

Patient 5 10p14-10pter view

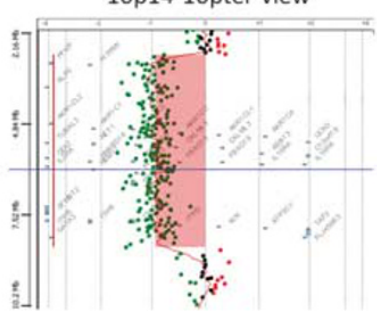

Patient 2

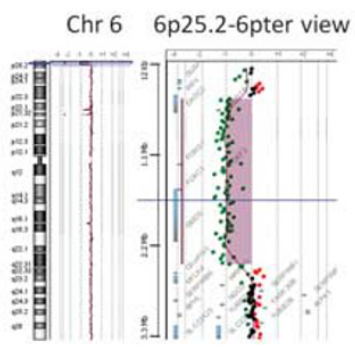

Patient 7

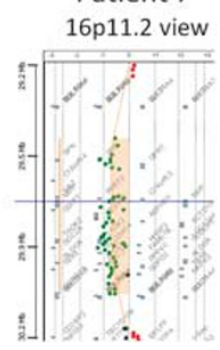

Patient 3

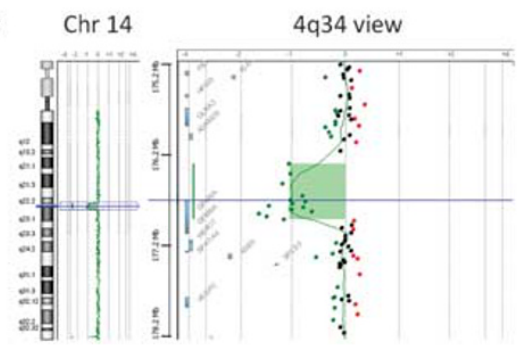

Patient 8 16p11.2 view

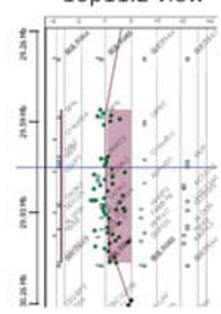

Patient 9

11p15.4 view

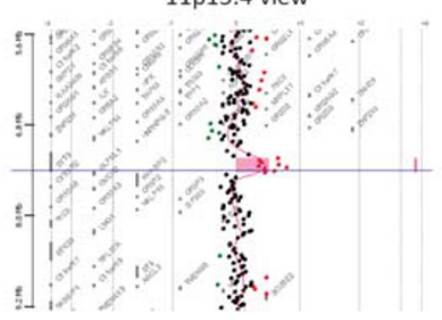

Patient 4

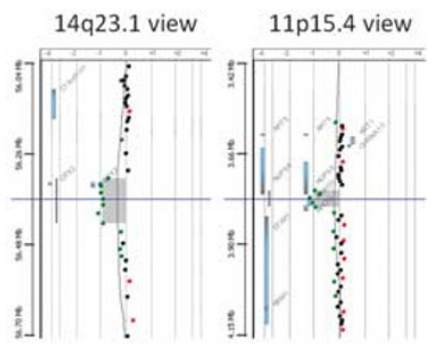

Patient 10

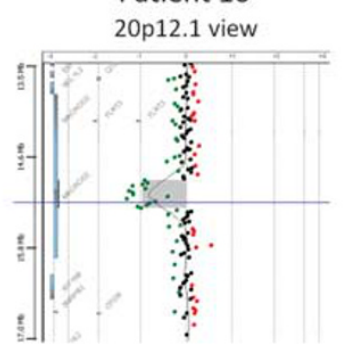

Figure 1 Possibly pathogenic chromosomal anomalies identified in patients with syndromal ODAs. Chromosome views and/or gene views of the affected chromosome or chromosome band produced by the Agilent CGH Analytics software and showing the aberrant region, which is highlighted in colour. On gene views, the dots correspond to the array targets, arranged on the $y$ axis according to their genomic position and on the $x$ axis according to their log2 intensity ratio value.

and maternally derived for patient no. 7. In patient no. 9, the inherited 11p15.4 duplication involving genes not known to be associated with disease was interpreted as possibly pathogenic because the pattern of family segregation suggested an autosomal dominant defect with incomplete penetrance and variable expressivity (Figure 2, Family A). In patient no. 10, we provisionally classified the $360-\mathrm{kb}$ 20p12.1 deletion as possibly pathogenic, although no known diseaseassociated gene, microdeletion, or microduplication syndrome was found. We are seeking to obtain samples from the parents and sisters to clarify the clinical significance of this deletion. In a male patient (patient no. 11) with an Xq25 deletion involving genes not known to be associated with disease and inherited from a healthy mother, the family study identified this Xq25 deletion in an asymptomatic maternal uncle and was therefore probably a non-pathogenic variant (Figure 2, family B, and Table 1). In three patients (patient no. 12, 13, and 14), DNA copy alterations were inherited from a phenotypically normal parent with no family history of ODA and were interpreted as likely non-pathogenic variants (Table 1). All 14 patients have been submitted for registration in the DECIPHER database (http://www. sanger.ac.uk/PostGenomics/decipher/). 

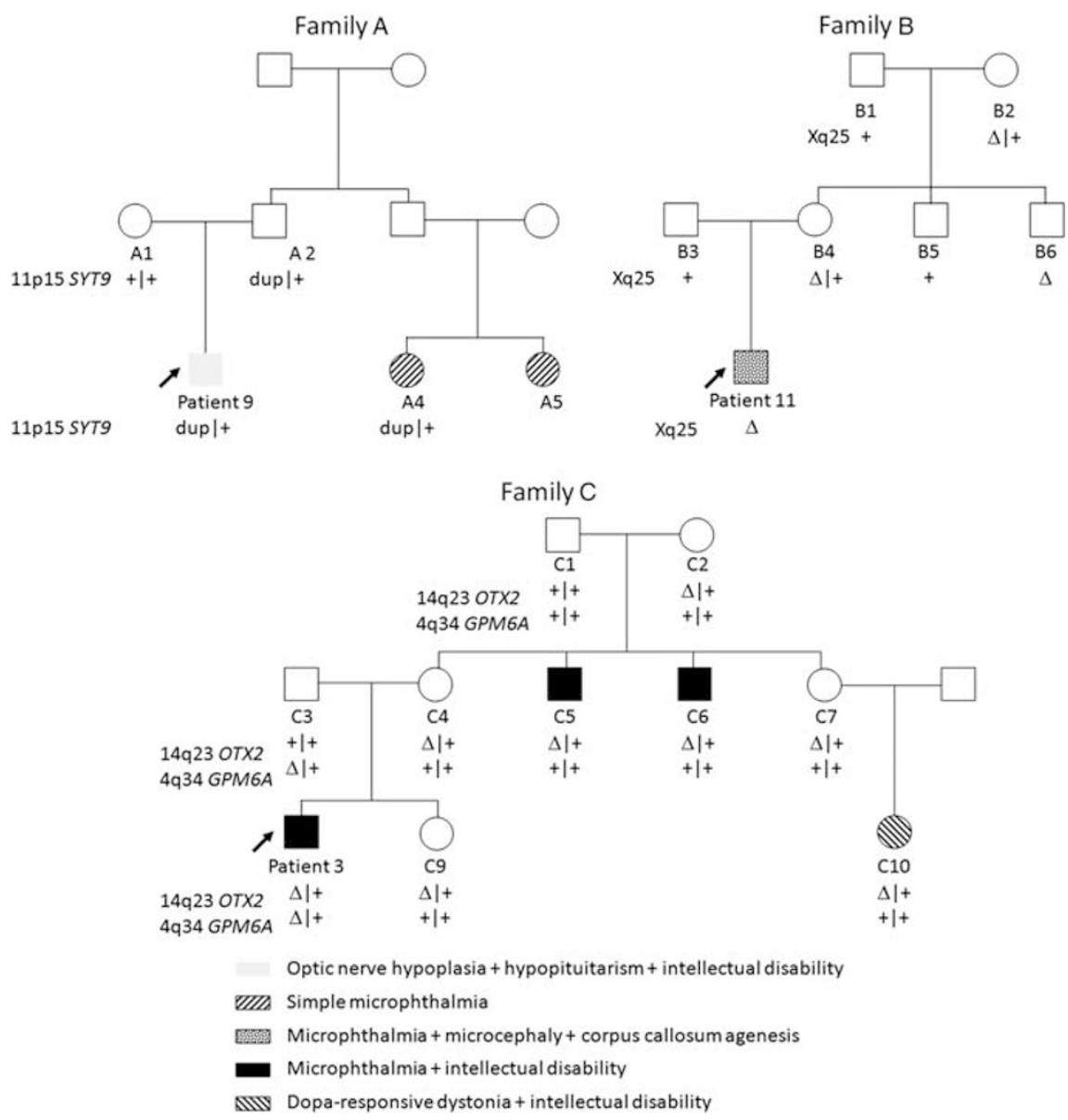

Figure 2 Family segregation of possibly pathogenic chromosomal anomalies. Family studies were performed for patient no. 9 (family A), 11 (family B), and 3 (family C). In family A, segregation of the $11 \mathrm{p} 15$ duplication was consistent with an autosomal dominant disorder with incomplete penetrance and variable expression. In family B, the Xq25 deletion was present in an asymptomatic maternal uncle, suggesting that it was a non-pathogenic variant devoid of clinical significance. Family $\mathrm{C}$ demonstrates the incomplete penetrance and variable expressivity of phenotypes associated with OTX2 defects. The additional paternally inherited imbalance in patient no. 3 suggests that GPM6A haploinsufficiency may modify the phenotype associated with OTX2 defects. dup, duplication; $\Delta$, deletion; +, wild type.

\section{Clinical data of patients with clinically relevant chromosomal abnormalities}

Patient no. 1. This girl with a $6 \mathrm{p} 25$ deletion encompassing the FOXC1 gene was referred to a clinical geneticist at 1 month of age for dilated cardiomyopathy and facial dysmorphism. She was the first and only child of healthy non-consanguineous parents. The pregnancy was uneventful. She was born at 39 weeks of gestation after a normal vaginal delivery. At birth, she had normal values for weight $(2845 \mathrm{~g})$, length $(47.5 \mathrm{~cm})$, and occipitofrontal circumference $(34 \mathrm{~cm})$. Dysmorphic features included a prominent forehead, hypertelorism, down-slanting palpebral fissures, a broad nasal bridge, and ocular proptosis. The combination of dilated cardiomyopathy and facial dysmorphism prompted further investigations. The conventional cytogenetic analysis was normal. The ophthalmologist found bilateral Axenfeld's anomaly with iridocorneal adhesions and corectopia of the right eye. Magnetic resonance imaging (MRI) of the brain showed a short corpus callosum and mega cisterna magna. During follow-up, mild motor delay developed and the cardiomyopathy remained stable. At last follow-up, she was 2 years of age and unable to walk alone; her weight was $10.2 \mathrm{~kg}(-1.5 \mathrm{SD})$, her height $81 \mathrm{~cm}(-1 \mathrm{SD})$, and her OFC $46 \mathrm{~cm}(-1 \mathrm{SD})$.
Patient no. 2. This 27-year-old man with a 6p25 deletion encompassing the FOXC1 gene was born to non-consanguineous parents. He had Rieger syndrome with low vision and bilateral Rieger ocular anomaly (iris hypoplasia, iridocorneal adhesions, and posterior embryotoxon) complicated by bilateral glaucoma. Other abnormalities were hypotrophy, hypodontia, and autistic spectrum disorder. Cerebral MRI could not be performed.

Patient no. 3. This 24-year-old male belonging to family C (Figure 2) and his two maternal uncles (C5 and C6) had various combinations of colobomatous microphthalmia, palate anomalies, facial dysmorphism, renal malformation, microcephaly, and intellectual disability. They had a $14 \mathrm{q} 23$ deletion encompassing the OTX2 gene. The carrier females had mild features (strabismus, nystagmus, low-normal intelligence, and speech problems), except one (C7), who was asymptomatic. Cousin $\mathrm{C} 10$ had developmental delay, strabismus, marked scoliosis, mild dysmorphism, and dopa-responsive dystonia. No other family members carrying the deletion had neurological symptoms suggesting dystonia.

Patient no. 4. This 14 year-old girl with a $14 \mathrm{q} 23$ deletion encompassing the OTX2 gene was the first of two children of healthy 
non-consanguineous parents. There was no family history of ODA. She was born at full term after a normal vaginal delivery. Her birth weight $(2400 \mathrm{~g})$ and length $(46 \mathrm{~cm})$ were at $-2 \mathrm{SD}$ (third percentile). She had severe bilateral microphthalmia, facial dysmorphism (prominent forehead and depressed nasal bridge), and developmental delay. When she was 4 years old, her weight was $10 \mathrm{~kg}(-3 \mathrm{SD})$, her height was $87 \mathrm{~cm}(-3.5 \mathrm{SD})$, and her skeletal bone age was 2 years. Investigations showed growth hormone deficiency and she was started on growth hormone replacement therapy. Brain MRI showed anterior pituitary hypoplasia and an ectopic posterior pituitary gland. At 10 years of age, after more than 5 years of growth hormone treatment, her weight was $21 \mathrm{~kg}(-2 \mathrm{SD})$ and her height $124 \mathrm{~cm}(-2 \mathrm{SD})$.

Patient no. 5. This 9-year-old boy with $10 \mathrm{p} 14 \mathrm{p} 15$ deletion was the first and only child of healthy non-consanguineous parents. He was born at full term after a normal vaginal delivery, with growth parameters at $-2 \mathrm{SD}$ : weight $2380 \mathrm{~g}$ (fifth percentile); length $45 \mathrm{~cm}$ (third percentile), and OFC $32 \mathrm{~cm}$ (fifth percentile). He had multiple birth defects consisting of choanal atresia, bilateral coloboma of the iris, malrotation of the midgut, grade III vesicoureteral reflux, facial dysmorphism (blepharophimosis with down-slanting palpebral fissures), and microcephaly (OFC at $-4 \mathrm{SD}$ ). During follow-up, epilepsy and developmental delay were diagnosed, followed by bilateral hearing impairment and hypoparathyroidism.

Patient no. 6. This 9-year-old boy previously described by Schiff et al ${ }^{11}$ as patient $\mathrm{D}$ had a $17 \mathrm{p} 13.3$. deletion. He was the second child of healthy non-consanguineous parents. He had prenatal-onset growth retardation, bilateral chorioretinal coloboma with lens coloboma and right microcornea, developmental delay, and facial dysmorphism (large face, hypertelorism, down-slanting palpebral fissures, ptosis, short nose, pointed chin, and everted lower lip). Multidrug-resistant epilepsy developed during follow-up.

Patient no. 7. This 4-year-old girl with a 16p11.2 deletion had septooptic dysplasia, with pituitary deficiency and intellectual disability. The ophthalmological examination was abnormal, with colobomatous microphthalmia of the left eye, persistent hyperplastic primary vitreous, and cataract of right eye. Brain MRI showed agenesis of the septum pellucidum and olfactory bulb and hypoplasia of the optic tract.

Patient no. 8. This 6-year-old boy with a $16 \mathrm{p} 11.2$ deletion was the second of two children of healthy non-consanguineous parents. There was no family history of ODA. He was born at full term after a normal vaginal delivery, with growth parameters at -2 SD: weight $2735 \mathrm{~g}$ (5-10th percentile); length $48 \mathrm{~cm}$ (10th percentile); and OFC $33 \mathrm{~cm}$ (10th percentile). Congenital nasolacrimal duct obstruction was repaired surgically. Chorioretinal coloboma with microcornea was found in the left eye; the right eye was normal. During follow-up, mild psychomotor delay with a speech defect developed. Brain MRI was not performed.

Patient no. 9. This 10-year-old boy with a $11 \mathrm{p} 15.4$ duplication was the first and only child of healthy non-consanguineous parents and belonged to family A (Figure 2). He had optic nerve hypoplasia, growth hormone and TSH deficiency, sleep disorder, and intellectual disability. Brain MRI showed anterior pituitary hypoplasia and ectopia of the posterior pituitary gland without other septal anomalies. His two paternal female cousins had simple microphthalmia.

Patient no. 10. This girl with a 20p12.1 deletion was the first-born child of healthy non-consanguineous parents. Abnormalities at birth consisted of bilateral anophthalmia, cleft lip and palate, deafness, and bilateral inguinal hernia. Her psychomotor development and stature were normal. She had a sister with unilateral microphthalmia and cleft lip and palate.

\section{DISCUSSION}

Using whole-genome array-CGH, we found 10 potentially pathogenic chromosome imbalances among 65 patients with ODAs. This diagnostic yield of $15 \%$ is consistent with the findings from oligonucleotide-based array studies in patients with unexplained intellectual disability or developmental delay. ${ }^{12,13}$ Our study's arrays resolutions are theoretically lower than those used in the two previous array-CGH studies of patients with ODA. ${ }^{8,9}$ However, our diagnostic yield is similar or better. This is certainly explained by differences in the inclusion criteria; unlike us, these two studies included patients with isolated ODAs.

The chromosomal abnormalities identified in patient no. 1-8 were considered causal. In four patients (no. 1-4), we found deletions encompassing a gene known to be involved in ocular development (FOXC1 or OTX2), in keeping with an earlier study. ${ }^{9}$ Adding our patients to those of this previous study, $8 \%$ of patients with ODAs had deletions encompassing OTX2 $(n=3)$, FOXC1 $(n=3)$, COH1 $(n=1)$, or PAX6 $(n=1)$. FOXC1 alterations (mutations or $6 \mathrm{p} 25.3$ deletions/ duplications) are involved in Axenfeld-Rieger anomaly or syndrome (MIM 601090, 602482, and 601631) and were recently shown to cause cerebellar vermis hypoplasia and to contribute to the Dandy-Walker continuum. $^{14}$ OTX2 deletions and heterozygous mutations cause syndromal micro-anophthalmia (MIM 600037 and 610125) ${ }^{15}$ and developmental anomalies of the pituitary gland, ${ }^{16}$ and OTX2 mutations can cause septo-optic dysplasia. ${ }^{17}$ Family $\mathrm{C}$ and several previously described families were reported with OTX2 alterations in patients presenting micro-anophthalmia and/or pituitary deficiency but inherited from an asymptomatic parent, ${ }^{9,16,18,19}$ suggesting the incomplete penetrance of OTX2-associated phenotypes. Therefore, a CNV encompassing OTX2 transmitted by an unaffected parent can be pathogenic for his child. Intrafamilial heterogeneity of OTX2 defects may be explained, in some cases, by an additional imbalance. The GPM6A gene included in the paternally inherited $610-\mathrm{kb}$ deletion at $4 \mathrm{q} 34$ of our patient no. 3 and the RHOG gene in the $11 \mathrm{p} 15$ deletion of patient no. 4 have both been implicated in neurite outgrowth. ${ }^{20,21}$ RHO-ROCK signalling is involved in regulating the microfilament system, which is a key regulator of epithelial morphogenesis ${ }^{22}$ and controls the early phases of optic cup morphogenesis. ${ }^{23}$ These data support a role for RHOG in ODAs. We therefore suggest that RHOG haploinsufficiency may modulate the OTX2-related phenotype of patient no. 4.

Patient no. 5 and no. 6 carry pathogenic deletions that are not classically associated with ODAs. In both patients, the extraocular phenotype is consistent with the deletion. Their ODA could be related to a separate unknown cause or explained by the incomplete penetrance of an unknown ocular developmental gene included in their deletion. In patient no. 5, the deletion includes GATA3, which is involved in the HDR syndrome (hypoparathyroidism, deafness, and renal dysplasia syndrome, MIM 146255), and the phenotype is consistent with HDR syndrome. The additional anomalies are possibly due to the other genes included in the large 10p14p15 deletion in this patient. In keeping with earlier data, ${ }^{11,24}$ ocular coloboma was a feature in our patient no. 6 , pointing to a critical $0.67-\mathrm{Mb}$ region for coloboma, with incomplete penetrance.

CNVs associated with partially penetrant phenotypes raise challenges, as seen also in our patient no. 7 and 8, who had a 16 p11.2 deletion. The 16p11.2 deletion syndrome (OMIM 611913) has been 
described in at least 100 individuals. The common deleted or duplicated region is $555 \mathrm{~kb}$ in length and is flanked by two low copy repeats of about $147 \mathrm{~kb}$, suggesting that its pathogenic effects may be mediated by non-allelic homologous recombination. Recurrent microdeletions and microduplications at 16p11.2 have been shown to confer susceptibility to autistic spectrum disorder and have been identified in up to $1 \%$ of patients with this diagnosis. ${ }^{25}$ The $16 \mathrm{p} 11.2$ deletion frequently co-segregates with severe early-onset obesity. ${ }^{26,27}$ ODA is not a common feature of $16 \mathrm{p} 11.2$ deletions. In a neonate with multiple anomalies, including right ocular coloboma and chorioretinitis, array-CGH detected a 16p11.2 deletion on the G-banded karyotype; the size of the deletion was not estimated, and the break points were not reported. ${ }^{28}$ More recently, microphthalmia and optic nerve coloboma were reported in a patient with a de novo $16 \mathrm{p} 11.2$ microdeletion. ${ }^{29}$ Our finding of a $16 \mathrm{p} 11.2$ deletion in two patients with ODAs supports a causal link between 16p11.2 haploinsufficiency and low-penetrance coloboma. A less likely hypothesis is that of an autosomal recessive disorder with loss of one of the alleles, allowing the mutation-carrying allele to cause the ODA. In this chromosomal region, SEZ6L2 may be involved in ocular development, as it is expressed in the forebrain during development ${ }^{30}$ as well as in the eye (BioGPS, http://biogps.gnf.org/; GeneHub-GEPIS, http://www.cgl. ucsf.edu/Research/genentech/genehub-gepis/genehub-gepis-search.html). Further studies are needed to evaluate the potential role for SEZ6L2 or another $16 \mathrm{p} 11.2$ gene in ocular development.

The clinical relevance of the CNV detected by array-CGH was unclear in two of our patients. Criteria for pathogenic CNVs, as opposed to non-pathogenic CNVs, have been suggested (see Table 1 of Lee et $a l^{31}$. Nevertheless, it may be difficult to draw a definitive conclusion, ${ }^{7,32}$ most notably when samples cannot be obtained from both parents (and, in some cases, other family members). Unavailability of family samples is probably the greatest challenge raised by the use of array-CGH as a routine diagnostic tool. In our patient no. 10 , the absence of family samples hindered our ability to interpret the $360-\mathrm{kb}$ deletion at 20p12.1 in exon 6 and intron 6 of MACROD2. This deletion is not registered among the non-pathogenic CNVs in the DGV database. The function of MACROD2 (previously c20orf133) is unknown. Genome-wide association studies found significant associations of MACROD2 single-nucleotide polymorphisms with autistic spectrum disorder, ${ }^{33}$ brain infarction, ${ }^{34}$ and brain volume in multiple sclerosis. ${ }^{35}$ The $360-\mathrm{kb}$ deletion is located close to a hotspot of de novo and inherited $\mathrm{CNVs}$ with variable and non-recurrent break points, involving exon 5 and/or intron 5 of MACROD2. CNVs of this hotspot were found in control individuals in the DGV database and in individuals with schizophrenia, ${ }^{36}$ holoprosencephaly, ${ }^{37}$ and Kabuki syndrome, ${ }^{38,39}$ suggesting limited or absent clinical relevance. ${ }^{40}$ The phenotype in patient no. 10 does not resemble Kabuki syndrome. These facts argue against a role for MACROD2 in ODAs.

The clinical relevance of the $11 \mathrm{p} 15$ microduplication detected in patient no. 9 is uncertain. This 180 -kb duplication partially encompasses two genes, SYT9 (OMIM 613528) and OLFML1. Confirmation using qPCR cannot distinguish a tandem/inverted duplication from an insertion at a distance. The SYT9 gene is specifically expressed in the mouse brain and may have a role in calcium-sensitive synaptic neurotransmitter release, ${ }^{41}$ but neither SYT9 nor OLFLM1 is known to be associated with clinical disease. The 11p15 duplication was also found in a cousin of patient no. 9 who has a different ODA. Microanophthalmia, optic nerve hypoplasia, and hypopituitarism belong to the growing spectrum of anomalies known to occur in septo-optic dysplasia. ${ }^{17}$ The pattern of family segregation is consistent with an autosomal dominant defect with incomplete penetrance and variable expression. In the absence of similar cases, we can suggest, but not confirm, that this variant is pathogenic.

In our study, two-thirds of the patients were males, in keeping with the known male predominance among patients with ODA (and intellectual disability). No pathogenic CNVs were found in the $\mathrm{X}$ chromosome in our study or in earlier work. Therefore, the male preponderance can probably not be ascribed to genomic rearrangements involving developmental genes on the $\mathrm{X}$ chromosome. However, we cannot rule out inadequate distribution of oligonucleotide probes on the $\mathrm{X}$ chromosome.

In conclusion, our results emphasise the benefits of whole-genome array-CGH for the diagnosis of ODA syndromes. Detecting the genetic defect has important consequences for genetic counselling of the families and follow-up of the patients. Detailed molecular analysis of the rearranged regions may help to identify the genes involved in ocular development.

\section{CONFLICT OF INTEREST}

The authors declare no conflict of interest.

\section{ACKNOWLEDGEMENTS}

We thank the patients and their families who participated in this study and the medical staff involved in the diagnosis of syndromal ocular development anomalies.

1 Fitzpatrick DR, van Heyningen V: Developmental eye disorders. Curr Opin Genet Dev 2005; 15: 348-353.

2 Hornby SJ, Gilbert CE, Rahi JK et al: Regional variation in blindness in children due to microphthalmos, anophthalmos and coloboma. Ophthalmic Epidemiol 2000; 7: 127-138.

3 Morrison D, FitzPatrick D, Hanson I et al: National study of microphthalmia, anophthalmia, and coloboma (MAC) in Scotland: investigation of genetic aetiology. J Med Genet 2002; 39: 16-22.

4 Mihelec M, St Heaps L, Flaherty M et al: Chromosomal rearrangements and novel genes in disorders of eye development, cataract and glaucoma. Twin Res Hum Genet 2008; 11: 412-421.

5 Kallen B, Tornqvist K: The epidemiology of anophthalmia and microphthalmia in Sweden. Eur J Epidemiol 2005; 20: 345-350.

6 Stoll C, Alembik Y, Dott B, Roth MP: Congenital eye malformations in 212,479 consecutive births. Ann Genet 1997; 40: 122-128.

7 Lee C, Scherer SW: The clinical context of copy number variation in the human genome. Expert Rev Mol Med 2010; 12: e8.

8 Raca G, Jackson CA, Kucinskas L et al: Array comparative genomic hybridization analysis in patients with anophthalmia, microphthalmia, and coloboma. Genet Med 2011; 13: 437-442.

9 Balikova I, de Ravel T, Ayuso C et al: High frequency of submicroscopic chromosomal deletions in patients with idiopathic congenital eye malformations. Am J Ophthalmol 2011; 151: 1087-1094, e1045.

10 Winer J, Jung CK, Shackel I, Williams PM: Development and validation of real-time quantitative reverse transcriptase-polymerase chain reaction for monitoring gene expression in cardiac myocytes in vitro. Anal Biochem 1999; 270: 41-49.

11 Schiff M, Delahaye A, Andrieux J et al: Further delineation of the 17p13.3 microdeletion involving YWHAE but distal to PAFAH1B1: four additional patients. Eur J Med Genet 2010; 53: 303-308.

12 Koolen DA, Pfundt R, de Leeuw N et al: Genomic microarrays in mental retardation: a practical workflow for diagnostic applications. Hum Mutat 2009; 30: 283-292.

13 Xiang B, Zhu H, Shen Y et al: Genome-wide oligonucleotide array comparative genomic hybridization for etiological diagnosis of mental retardation: a multicenter experience of 1499 clinical cases. J Mol Diagn 2010; 12: 204-212.

14 Aldinger KA, Lehmann OJ, Hudgins $L$ et al: FOXC1 is required for normal cerebellar development and is a major contributor to chromosome 6p25.3 Dandy-Walker malformation. Nat Genet 2009; 41: 1037-1042.

15 Nolen LD, Amor D, Haywood A et al: Deletion at 14q22-23 indicates a contiguous gene syndrome comprising anophthalmia, pituitary hypoplasia, and ear anomalies. $\mathrm{Am} \mathrm{J}$ Med Genet A 2006; 140: 1711-1718.

16 Dateki S, Kosaka K, Hasegawa K et al: Heterozygous orthodenticle homeobox 2 mutations are associated with variable pituitary phenotype. J Clin Endocrinol Metab 2010; 95: 756-764.

17 McCabe MJ, Alatzoglou KS, Dattani MT: Septo-optic dysplasia and other midline defects: the role of transcription factors: HESX1 and beyond. Best Pract Res Clin Endocrinol Metab 2011; 25: 115-124. 
18 Ragge NK, Brown AG, Poloschek CM et al: Heterozygous mutations of OTX2 cause severe ocular malformations. Am J Hum Genet 2005; 76: 1008-1022.

19 Schilter KF, Schneider A, Bardakjian T et al: OTX2 microphthalmia syndrome: four novel mutations and delineation of a phenotype. Clin Genet 2011; 79: 158-168.

20 Alfonso J, Fernandez ME, Cooper B, Flugge G, Frasch AC: The stress-regulated protein M6a is a key modulator for neurite outgrowth and filopodium/spine formation. Proc Natl Acad Sci USA 2005; 102: 17196-17201.

21 Katoh H, Negishi M: RhoG activates Rac1 by direct interaction with the Dock180binding protein Elmo. Nature 2003; 424: 461-464.

22 Sawyer JM, Harrell JR, Shemer G, Sullivan-Brown J, Roh-Johnson M, Goldstein B: Apical constriction: a cell shape change that can drive morphogenesis. Dev Biol 2010; 341: 5-19.

23 Eiraku M, Takata N, Ishibashi $\mathrm{H}$ et al: Self-organizing optic-cup morphogenesis in three-dimensional culture. Nature 2011; 472: 51-56.

24 Nagamani SC, Zhang F, Shchelochkov OA et al: Microdeletions including YWHAE in the Miller-Dieker syndrome region on chromosome $17 \mathrm{p} 13.3$ result in facial dysmorphisms, growth restriction, and cognitive impairment. J Med Genet 2009; 46: 825-833.

25 Fernandez BA, Roberts W, Chung B et al: Phenotypic spectrum associated with de novo and inherited deletions and duplications at $16 \mathrm{p} 11.2$ in individuals ascertained for diagnosis of autism spectrum disorder. J Med Genet 2010; 47: 195-203.

26 Bochukova EG, Huang N, Keogh J et al: Large, rare chromosomal deletions associated with severe early-onset obesity. Nature 2010; 463: 666-670.

27 Walters RG, Jacquemont S, Valsesia A et al: A new highly penetrant form of obesity due to deletions on chromosome 16p11.2. Nature 2010; 463: 671-675.

28 Hernando C, Plaja A, Rigola MA et al: Comparative genomic hybridisation shows a partial de novo deletion $16 \mathrm{p} 11.2$ in a neonate with multiple congenital malformations. J Med Genet 2002; 39: E24.

29 Bardakjian TM, Kwok S, Slavotinek AM, Schneider AS: Clinical report of microphthalmia and optic nerve coloboma associated with a de novo microdeletion of chromosome 16p11.2. Am J Med Genet A 2010; 152A: 3120-3123.
30 Kumar RA, Marshall CR, Badner JA et al: Association and mutation analyses of 16p11.2 autism candidate genes. PLoS One 2009; 4: e4582.

31 Lee C, lafrate AJ, Brothman AR: Copy number variations and clinical cytogenetic diagnosis of constitutional disorders. Nat Genet 2007; 39: S48-S54.

32 Rodriguez-Revenga L, Mila M, Rosenberg C, Lamb A, Lee C: Structural variation in the human genome: the impact of copy number variants on clinical diagnosis. Genet Med 2007; 9: 600-606.

33 Anney R, Klei L, Pinto D et al: A genome-wide scan for common alleles affecting risk for autism. Hum Mol Genet 2010; 19: 4072-4082.

34 Debette S, Bis JC, Fornage M et al: Genome-wide association studies of MRI-defined brain infarcts: meta-analysis from the CHARGE Consortium. Stroke 2010; 41: 210-217.

35 Baranzini SE, Wang J, Gibson RA et al: Genome-wide association analysis of susceptibility and clinical phenotype in multiple sclerosis. Hum Mol Genet 2009; 18: 767-778.

36 Xu B, Woodroffe A, Rodriguez-Murillo $L$ et al: Elucidating the genetic architecture of familial schizophrenia using rare copy number variant and linkage scans. Proc Natl Acad Sci USA 2009; 106: 16746-16751.

37 Bendavid C, Rochard L, Dubourg C et al: Array-CGH analysis indicates a high prevalence of genomic rearrangements in holoprosencephaly: an updated map of candidate loci. Hum Mutat 2009; 30: 1175-1182.

38 Maas NM, Van de Putte T, Melotte $\mathrm{C}$ et al: The $\mathrm{C} 20$ orf 133 gene is disrupted in a patient with Kabuki syndrome. J Med Genet 2007; 44: 562-569.

39 Kuniba H, Yoshiura K, Kondoh $\mathrm{T}$ et al: Molecular karyotyping in 17 patients and mutation screening in 41 patients with Kabuki syndrome. J Hum Genet 2009; 54: 304-309.

40 Bradley WE, Raelson JV, Dubois DY et al: Hotspots of large rare deletions in the human genome. PLoS One 2010; 5: e9401.

41 Xu J, Mashimo T, Sudhof TC: Synaptotagmin-1, -2, and -9: $\mathrm{Ca}(2+)$ sensors for fast release that specify distinct presynaptic properties in subsets of neurons. Neuron 2007; 54: 567-581.

Supplementary Information accompanies the paper on European Journal of Human Genetics website (http://www.nature.com/ejhg) 